\title{
HYPERREFLEXIVITY AND A DUAL PRODUCT CONSTRUCTION ${ }^{1}$
}

\author{
BY
}

DAVID R. LARSON

\begin{abstract}
We show that an example of a nonhyperreflexive CSL algebra recently constructed by Davidson and Power is a special case of a general and natural reflexive subspace construction. Completely different techniques of proof are needed because of absence of symmetry. It is proven that if $\mathscr{S}$ and $\mathscr{T}$ are reflexive proper linear subspaces of operators acting on a separable Hilbert space, then the hyperreflexivity constant of $\left(\mathscr{S}_{\perp} \otimes \mathscr{T}_{\perp}\right)^{\perp}$ is at least as great as the product of the constants of $\mathscr{S}$ and $\mathscr{T}$.
\end{abstract}

This paper was inspired by the interesting "key example" in the recent paper [2] by Davidson and Power in which a nonhyperreflexive CSL algebra was constructed. In an attempt to completely understand this result we obtained a distance constant inequality of a more general nature, which we present here.

Let $H, K$ be separable Hilbert spaces-finite or infinite dimensional-and let $\mathscr{S}, \mathscr{T}$ be linear subspaces of $L(H), L(K)$, which are reflexive in the LoginovShulman sense. ( $\mathscr{S}$ is reflexive iff whenever $T \in L(H)$ is such that $T x \in[S x]$, $x \in H$, then $T \in \mathscr{S}$, where [ $]$ means closure.) Let $\mathscr{K}(\mathscr{S}), \mathscr{K}(\mathscr{T})$ be the constants of hyperreflexivity of $\mathscr{S}$ and $\mathscr{T}$ as defined in [4]. We recall that a subspace $\mathscr{S}$ of $L(H)$ is hyperreflexive if there is a constant $C$ such that for operators $T$ in $L(H)$,

$$
d(T, \mathscr{S}) \leqslant C \sup \left\{\left\|P^{\perp} T Q\right\|: P, Q \text { are projections with } P^{\perp} \mathscr{S} Q=0\right\},
$$

and the optimal constant is denoted $\mathscr{K}(\mathscr{S})$. If $\mathscr{S}$ is reflexive but not hyperreflexive, then we define $\mathscr{K}(\mathscr{S})=+\infty$. We make use of preannihilator techniques, and refer the reader to $[1,4,5,7]$ for details. As shown in [4], the reflexive subspaces of $L(H)$ are precisely those for which the preannihilator in $\mathscr{L}_{*} \equiv C_{1}$ is the $\|\cdot\|_{1}$-closed linear span of rank $\leqslant 1$ operators, where $\|\cdot\|_{1}$ denotes trace-class norm. Since $\mathscr{S}_{\perp}, \mathscr{T}_{\perp}$ are generated by rank $\leqslant 1$ operators, so is the tensor product of preannihilators $\mathscr{S}_{\perp} \otimes \mathscr{T}_{\perp}$. By this we mean the $\|\cdot\|_{1}$-closed linear subspace of the ideal of trace-class operators on $L(H \otimes K)$ generated by the elementary tensors $\left\{f \otimes g: f \in \mathscr{S}_{\perp}\right.$, $\left.g \in \mathscr{T}_{\perp}\right\}$, where $H \otimes K$ denotes the usual tensor product Hilbert space. (When we write $\mathscr{S} \otimes \mathscr{T}$, we will mean the $\sigma$-weakly closed linear subspace of $L(H \otimes K)$ generated by $\{S \otimes T: S \in \mathscr{S}, T \in \mathscr{T}\}$.) Thus the annihilator

$$
\left(\mathscr{S}_{\perp} \otimes \mathscr{T}_{\perp}\right)^{\perp}=\left\{A \in L(H \otimes K): \operatorname{Tr}(A h)=0, h \in \mathscr{S}_{\perp} \otimes \mathscr{T}_{\perp}\right\}
$$

Received by the editors April 15, 1985.

1980 Mathematics Subject Classification. Primary 47D25: Secondary 47A15. 46L10.

Key words and phrases. Reflexive operator algebra, hyperreflexive, tensor product, dual product. distance constant, preannihilator.

${ }^{1}$ This work was partially supported by NSF grant MCS-8301740. 
is a reflexive subspace of $L(H \otimes K)$. We will call this the dual product of $\mathscr{S}$ and $\mathscr{T}$ since it is in a sense dual to the usual tensor product, and will adopt the notation $\mathscr{S} * \mathscr{T}=\left(\mathscr{S}_{\perp} \otimes \mathscr{T}_{\perp}\right)^{\perp}$. We extend this term, and notation, to arbitrary $\sigma$-weakly closed subspaces.

For reflexive $\mathscr{S}$ and $\mathscr{T}$, Theorem 8 states that $\mathscr{S} * \mathscr{T}$ is the smallest reflexive subspace containing $\mathscr{S} \otimes L(K)+L(H) \otimes \mathscr{T}$. In special cases (and perhaps in general) this coincides with the $\sigma$-weak closure of $\mathscr{S} \otimes L(K)+L(H) \otimes \mathscr{T}$. This is the case in finite dimensions (Proposition 1).

It is clear that for subspaces $\mathscr{S}_{i}$ we have $\left(\mathscr{S}_{1} * \mathscr{S}_{2}\right) * \mathscr{S}_{3}=\mathscr{S}_{1} *\left(\mathscr{S}_{2} * \mathscr{S}_{3}\right)$ (or rather, equivalence), so we may drop parentheses with no ambiguity. An $n$-fold dual product $\mathscr{S}_{1} * \cdots * \mathscr{S}_{n}$ will have the $n$-fold tensor product $\left(\mathscr{S}_{1}\right)_{\perp} \otimes \cdots \otimes\left(\mathscr{S}_{n}\right)_{\perp}$ as preannihilator.

The main result of this paper, Theorem 9 , states that the inequality $\mathscr{K}(\mathscr{S} * \mathscr{T}) \geqslant$ $\mathscr{K}(\mathscr{S}) \cdot \mathscr{K}(\mathscr{T})$ always holds for reflexive proper subspaces $\mathscr{S}, \mathscr{T}$. (If either $\mathscr{S}=L(H)$ or $\mathscr{T}=L(K)$, then $\mathscr{S} * \mathscr{T}=L(H \otimes K)$, so the inequality need not hold. These are the only exceptions.)

A special case arises when $\mathscr{D}$ is the algebra of $3 \times 3$ diagonal operators acting on a 3-dimensional Hilbert space. Then, since it is known (M. D. Choi, unpublished) that $\mathscr{K}(\mathscr{D}) \geqslant \sqrt{9 / 8}$, the $n$-fold dual product $\mathscr{D} * \cdots * \mathscr{D}$ has constant $\geqslant(9 / 8)^{n / 2}$. This is seen to be the Davidson-Power example. The subspace $\mathscr{D} * \cdots * \mathscr{D}$ is a bimodule over the $n$-fold tensor product $\mathscr{D} \otimes \cdots \otimes \mathscr{D}$, a m.a.s.a.; hence

$$
\left(\begin{array}{cc}
\mathscr{D} \otimes \cdots \otimes \mathscr{D} & \mathscr{D} * \cdots * \mathscr{D} \\
0 & \mathscr{D} \otimes \cdots \otimes \mathscr{D}
\end{array}\right)
$$

is a CSL algebra. It can be shown directly, as in [2], or via duality, as in Theorem 12, that the hyperreflexivity constant for this algebra is at least as great as that of $\mathscr{D} * \cdots * \mathscr{D}$. Theorem 9 can be viewed as a generalization of the induction step in the Davidson-Power construction. Since averaging techniques utilizing symmetry do not apply, proofs are necessarily different. Prior to their example, inequalities of this nature were not suspected.

We note that while $\mathscr{D}$ is an algebra, $\mathscr{D} * \mathscr{D}$ is not. Hence, analysis of multi-dualproducts such as $\mathscr{D} * \cdots * \mathscr{D}$ requires reflexive subspace theory. Also, we note that Propositions 1 and 3 are not used in the proofs of our main results, but are given for perspective on these.

The next lemma will be used repeatedly.

Lemma 0 . Let $\mathscr{S} \subseteq L(H), \mathscr{T} \subseteq L(K)$ be linear subspaces. Then $\mathscr{S} * \mathscr{T} \supseteq \mathscr{S} \otimes$ $L(K)+L(H) \otimes \mathscr{T}$.

Proof. If $f \in \mathscr{S}_{\perp}, g \in \mathscr{T}_{\perp}$, then for each $S \in \mathscr{S}, T \in \mathscr{T}, A \in L(H), B \in$ $L(K)$ we have

$$
\begin{aligned}
\operatorname{Tr}[(S \otimes B+A \otimes T)(f \otimes g)] & =\operatorname{Tr}[(s f) \otimes(B g)]+\operatorname{Tr}[(A f) \otimes(T g)] \\
& =\operatorname{Tr}(S f) \cdot \operatorname{Tr}(B g)+\operatorname{Tr}(A f) \cdot \operatorname{Tr}(T g)=0
\end{aligned}
$$

since $\operatorname{Tr}(S f)=0$ and $\operatorname{Tr}(T g)=0$. Since the operators $S \otimes B+A \otimes T$ generate $\mathscr{S} \otimes L(K)+L(H) \otimes \mathscr{T}$, and the operators $f \otimes g$ generate $\mathscr{S}_{\perp} \otimes \mathscr{T}_{\perp}=(\mathscr{S} * \mathscr{T})_{\perp}$, we conclude that $(\mathscr{S} * \mathscr{T})_{\perp} \subseteq(\mathscr{S} \otimes L(K)+L(H) \otimes \mathscr{T})_{\perp}$, and hence that $\mathscr{S} \otimes \mathscr{T}$ $\supseteq \mathscr{S} \otimes L(K)+L(H) \otimes \mathscr{T}$. 
Proposition 1. Let $H, K$ be finite dimensional Hilbert spaces, and let $\mathscr{S} \subseteq L(H)$, $\mathscr{T} \subseteq L(K)$ be linear subspaces. Then $\mathscr{S} * \mathscr{T}=\mathscr{S} \otimes L(K)+L(H) \otimes \mathscr{T}$.

Proof. Let $n_{1}, n_{2}$ be the dimensions of $H, K$, respectively, and let $m_{1}, m_{2}$ be the vector space dimensions of $\mathscr{S}, \mathscr{T}$, respectively. Then $\operatorname{dim}(L(H))=n_{1}^{2}, \operatorname{dim}(L(K))$ $=n_{2}^{2}, \operatorname{dim}\left(\mathscr{S}_{\perp}\right)=n_{1}^{2}-m_{1}$ and $\operatorname{dim}\left(\mathscr{T}_{\perp}\right)=n_{2}^{2}-m_{2}$. So $\operatorname{dim}\left(\mathscr{S}_{\perp} \otimes \mathscr{T}_{\perp}\right)=$ $\left(n_{1}^{2}-m_{1}\right)\left(n_{2}^{2}-m_{2}\right)$, and thus

$$
\operatorname{dim}(\mathscr{S} \otimes \mathscr{T})=n_{1}^{2} n_{2}^{2}-\left(n_{1}^{2}-m_{1}\right)\left(n_{2}^{2}-m_{2}\right)=n_{1}^{2} m_{2}+m_{1} n_{2}^{2}-m_{1} m_{2} .
$$

If $X, Y$ are finite dimensional vector spaces over $\mathbf{C}$, and if $X_{1} \subseteq X, Y_{1} \subseteq Y$ are linear subspaces, then $\left(X_{1} \otimes Y\right) \cap\left(X \otimes Y_{1}\right)=X_{1} \otimes Y_{1}$. Thus $(\mathscr{S} \otimes L(K)) \cap$ $(L(H) \otimes \mathscr{T})=\mathscr{S} \otimes \mathscr{T}$. So

$$
\begin{aligned}
\operatorname{dim}(\mathscr{S} \otimes L(K)+L & (H) \otimes \mathscr{T}) \\
& =\operatorname{dim}(\mathscr{S} \otimes L(K))+\operatorname{dim}(L(H) \otimes \mathscr{T})-\operatorname{dim}(\mathscr{S} \otimes \mathscr{T}) \\
& =m_{1} n_{2}^{2}+n_{1}^{2} m_{2}-m_{1} m_{2}=\operatorname{dim}(\mathscr{S} * \mathscr{T}) .
\end{aligned}
$$

So by Lemma 0 we must have $\mathscr{S} * \mathscr{T}=\mathscr{S} \otimes L(K)+L(H) \otimes \mathscr{T}$.

If $\mathscr{A} \subseteq L(H)$ is a reflexive algebra and $T \in L(H)$, the Arveson estimate for the distance from $T$ to $\mathscr{A}$ is $\alpha(T, \mathscr{A})=\sup \left\{\left\|P^{\perp} T P\right\|: P \in\right.$ lat $\left.\mathscr{A}\right\}$. For reflexive subspaces $\mathscr{S} \subseteq L(H)$ the estimate is defined [4] by $\alpha(T, \mathscr{S})=\sup \left\{\left\|P^{\perp} T Q\right\|: P, Q\right.$ are projections with $\left.P^{\perp} \mathscr{S} Q=0\right\}$. This agrees with the "PTP ${ }^{\perp}$ " formula when $I \in \mathscr{S}$. There is a "projection free" characterization of this estimate which proves useful. Let $d(\cdot, \cdot)$ denote distance.

Lemma 2. Let $\mathscr{S} \subseteq L(H)$ be a reflexive subspace. Then $\alpha(T, \mathscr{S})=$ $\sup \{d(T x, \mathscr{S} x): x \in H,\|x\|=1\}, T \in L(H)$.

Proof. We have $d(T x, \mathscr{S} x)=\left\|P^{\perp} T x\right\|$, where $P$ is the orthogonal projection onto $[\mathscr{S} x]$. Let $\|x\|=1$ and let $Q$ be the projection onto $\mathbf{C} x$. Then $\left\|P^{\perp} T x\right\|=$ $\left\|P^{\perp} T Q\right\|$. Since $P^{\perp} \mathscr{S} Q=0$, the inequality “ $\geqslant$ " follows.

Conversely, suppose $P, Q$ are projections with $P^{\perp} \mathscr{S} Q=0$. Let $\varepsilon>0$ be given, and choose $x \in Q H,\|x\|=1$, such that $\left\|P^{\perp} T x\right\| \geqslant\left\|P^{\perp} T Q\right\|-\varepsilon$. Then

$$
d(T x, \mathscr{S} x) \geqslant d(T x, P H)=\left\|P^{\perp} T x\right\| \geqslant\left\|P^{\perp} T Q\right\|-\varepsilon .
$$

Since $\varepsilon$ was arbitrary, we have $\left\|P^{\perp} T Q\right\| \leqslant \sup \{d(T, \mathscr{S} x): x \in H,\|x\|=1\}$. Taking the supremum over all pairs $\{P, Q\}$ with $P^{\perp} \mathscr{S} Q=0$ completes the proof.

Lemma 2 points out that only cyclic projections $P$ need be considered in distance estimate computations. Also, taking this as the definition permits natural extension of the concept to general normed linear spaces.

If $\mathscr{S} \neq L(H)$ is reflexive and $T \notin \mathscr{S}$, we write $\mathscr{K}(T, \mathscr{S})=d(T, \mathscr{S}) / \alpha(T, \mathscr{S})$. So $\mathscr{K}(\mathscr{S})=\sup \{\mathscr{K}(T, \mathscr{S}): T \in L(H), T \notin \mathscr{S}\}$. By convention $\mathscr{K}(L(H))=1 . \mathscr{S}$ is hyperreflexive if $\mathscr{K}(\mathscr{S})<\infty$, and is nonhyperreflexive otherwise.

We first give an initial generalization of the Davidson-Power induction step in which use is made of symmetry. The proof is more direct than that of our general result, so is included for perspective. 
Proposition 3. Let $\mathscr{S}$ be a reflexive subspace of $L(H)$, with $\mathscr{S} \neq L(H)$. Let

$$
\tilde{\mathscr{S}}=\left(\begin{array}{lll}
* & \mathscr{S} & \mathscr{S} \\
\mathscr{S} & * & \mathscr{S} \\
\mathscr{S} & \mathscr{S} & *
\end{array}\right)
$$

be the subspace of all $3 \times 3$ operator matrices with diagonal elements arbitrary and off-diagonal elements in $\mathscr{S}$. Then $\mathscr{K}(\tilde{\mathscr{S}}) \geqslant \sqrt{9 / 8} \cdot \mathscr{K}(\mathscr{S})$.

Proof. First, suppose $\mathscr{K}(\mathscr{S})$ is finite. Fix $\varepsilon>0$. Choose $T \in L(H)$ for which $\mathscr{K}(T, \mathscr{S}) \geqslant \mathscr{K}(\mathscr{S})-\varepsilon$. Let

$$
\tilde{T}=\left(\begin{array}{lll}
T & T & T \\
T & T & T \\
T & T & T
\end{array}\right) .
$$

The averaging technique used in the proof of Theorem 1.1 in [2] yields without modification that $d(\tilde{T}, \tilde{\mathscr{S}})=\frac{3}{2} \cdot d(T, \mathscr{S})$. We need only show that $\alpha(\tilde{T}, \tilde{\mathscr{S}})$ $\leqslant \sqrt{2} \alpha(T, \mathscr{S})$, for then

$$
\mathscr{K}(\tilde{\mathscr{S}}) \geqslant \mathscr{K}(\tilde{T}, \tilde{\mathscr{S}}) \geqslant \sqrt{9 / 8} \mathscr{K}(T, \mathscr{S})>\sqrt{9 / 8}(\mathscr{K}(\mathscr{S})-\varepsilon),
$$

and since $\varepsilon$ was arbitrary the desired inequality would follow.

To show that $\alpha(\tilde{T}, \tilde{\mathscr{S}}) \leqslant \sqrt{2} \alpha(T, \mathscr{S})$, it is useful to use Lemma 2. Let

$$
\tilde{x}=\left(\begin{array}{l}
x_{1} \\
x_{2} \\
x_{3}
\end{array}\right)
$$

be an arbitrary unit vector in $H \otimes H_{3}$. Then

$$
\tilde{T} \tilde{x}=\left(\begin{array}{c}
T z \\
T z \\
T z
\end{array}\right),
$$

where $z=x_{1}+x_{2}+x_{3}$. Descriptively, we have

$$
\tilde{\mathscr{S}} \tilde{x}=\left(\begin{array}{ccc}
* & \mathscr{S} & \mathscr{S} \\
\mathscr{S} & * & \mathscr{S} \\
\mathscr{S} & \mathscr{S} & *
\end{array}\right)\left(\begin{array}{l}
x_{1} \\
x_{2} \\
x_{3}
\end{array}\right)=\left(\begin{array}{l}
L(H) x_{1}+\mathscr{S} x_{2}+\mathscr{S} x_{3} \\
\mathscr{S} x_{1}+L(H) x_{2}+\mathscr{S} x_{3} \\
\mathscr{S} x_{1}+\mathscr{S} x_{2}+L(H) x_{3}
\end{array}\right) .
$$

We consider three cases:

(1) If neither $x_{1}, x_{2}$ nor $x_{3}=0$, then $\tilde{\mathscr{S}} \tilde{x}=H \otimes H_{3}$, so $d(\tilde{T} \tilde{x}, \tilde{\mathscr{S}} \tilde{x})=0$.

(2) If precisely one of $x_{1}, x_{2}, x_{3}$ is 0 , then without loss of generality we may assume $x_{1}=0$ by noting that $\tilde{\mathscr{S}}$ is invariant under the group of unitary transformations corresponding to permutation of basis vectors in $H_{3}$. We have $z=x_{2}+x_{3}$, and

$$
\tilde{\mathscr{S}} \tilde{x}=\left(\begin{array}{c}
\mathscr{S} x_{2}+\mathscr{S} x_{3} \\
L(H) \\
L(H)
\end{array}\right)
$$

so

$$
d(\tilde{T} \tilde{x}, \tilde{\mathscr{S}} \tilde{x})=d\left(T z, \mathscr{S} x_{2}+\mathscr{S} x_{3}\right) \leqslant d(T z, \mathscr{S} z)
$$


We have $\|z\| \leqslant \sqrt{2}$. If $z=0$, then $d(\tilde{T} \tilde{x}, \tilde{\mathscr{S}} \tilde{x})=0$. If $z \neq 0$, let $w=z /\|z\|$. Then

$$
d(\tilde{T} \tilde{x}, \tilde{\mathscr{S}} \tilde{x}) \leqslant \sqrt{2} d(T w, \mathscr{S} w) \leqslant \sqrt{2} \alpha(T, S),
$$

as desired.

(3) If precisely two of $x_{1}, x_{2}, x_{3}$ are 0 , via permutation as above, we may assume $x_{1}=x_{2}=0$. Then $z=x_{3}$, so $\|z\|=1$. We have

$$
\tilde{\mathscr{S}} \tilde{x}=\left(\begin{array}{c}
\mathscr{S}_{z} \\
\mathscr{S}_{z} \\
L(H)
\end{array}\right),
$$

so

$$
d(\tilde{T} \tilde{x}, \tilde{\mathscr{S}} \tilde{x})=\sqrt{2} d(T z, \mathscr{S} z) \leqslant \sqrt{2} \alpha(T, \mathscr{S}) .
$$

Now from cases (1)-(3) we have

$$
\begin{aligned}
\alpha(\tilde{T}, \tilde{\mathscr{S}}) & =\sup \left\{d(\tilde{T} \tilde{x}, \tilde{\mathscr{S}} \tilde{x}): \tilde{x} \in H \otimes H_{3},\|\tilde{x}\|=1\right\} \\
& \leqslant \sqrt{2} \alpha(T, \mathscr{S})
\end{aligned}
$$

as required. For the case $\mathscr{K}(\mathscr{S})=\infty$, let $n \geqslant 1$ be arbitrary and choose $T$ with $\mathscr{K}(T, \mathscr{S}) \geqslant n$. The same argument as above shows that $\mathscr{K}(\tilde{T}, \tilde{\mathscr{S}}) \geqslant \sqrt{9 / 8} n$. Hence $\mathscr{K}(\tilde{\mathscr{S}})=+\infty$.

A simple duality computation shows that the preannihilator of $\tilde{\mathscr{S}}$ in Proposition 3 has the form

$$
\tilde{\mathscr{S}}_{\perp}=\left(\begin{array}{ccc}
0 & \mathscr{S}_{\perp} & \mathscr{S}_{\perp} \\
\mathscr{S}_{\perp} & 0 & \mathscr{S}_{\perp} \\
\mathscr{S}_{\perp} & \mathscr{S}_{\perp} & 0
\end{array}\right),
$$

where $\mathscr{S}_{\perp}$ is the preannihilator of $\mathscr{S}$. The preannihilator of $\mathscr{D}_{3}$ has the form

$$
\left(\begin{array}{lll}
0 & * & * \\
* & 0 & * \\
* & * & 0
\end{array}\right)
$$

so $\tilde{\mathscr{S}}_{\perp}=\mathscr{S}_{\perp} \otimes\left(\mathscr{D}_{3}\right)_{\perp}$, and hence $\tilde{\mathscr{S}}=\left(\mathscr{S}_{\perp} \otimes\left(\mathscr{D}_{3}\right)_{\perp}\right)^{\perp}=\mathscr{S} * \mathscr{D}_{3}$. This suggests that a generalization is possible. The next proposition is used in place of an averaging technique.

Proposition 4. Let $\mathscr{S} \subseteq L(H), \mathscr{T} \subseteq L(K)$ be $\sigma$-weakly closed subspaces. If $A \in L(H)$ and $B \in L(K)$ are arbitrary, then $d(A \otimes B, \mathscr{S} * \mathscr{T})=d(A, \mathscr{S})$. $d(B, \mathscr{T})$.

Proof. Let $\mathscr{R}=\mathscr{S} \otimes L(K)+L(H) \otimes \mathscr{T}$. By Lemma 0 we have $\mathscr{R} \subseteq \mathscr{S} * \mathscr{T}$, so for each $S \in \mathscr{S}$ and $T \in \mathscr{T}$ we have $(A-S) \otimes(B-T)-A \otimes B \in \mathscr{R} \subseteq \mathscr{S} * \mathscr{T}$. Thus

$$
d(A \otimes B, \mathscr{S} * \mathscr{T})=d((A-S) \otimes(B-T), \mathscr{S} * \mathscr{T}) \leqslant\|A-S\| \cdot\|B-T\| .
$$

It follows that $d(A \otimes B, \mathscr{S} * \mathscr{T}) \leqslant d(A, \mathscr{S}) \cdot d(B, \mathscr{T})$. 
For the reverse inequality, let $\varepsilon>0$ be given and choose $f \in \mathscr{S}_{\perp}, g \in \mathscr{T}_{\perp}$ with $\|f\|_{1}=\|g\|_{1}=1$, such that $\operatorname{Tr}(A f)>d(A, \mathscr{S})-\varepsilon$, and $\operatorname{Tr}(B g)>d(B, \mathscr{T})-\varepsilon$. Let $h=f \otimes g$. We have

$$
\begin{aligned}
|\operatorname{Tr}[(A \otimes B) h]| & =|\operatorname{Tr}(A f \otimes B g)|=|\operatorname{Tr}(A f)| \cdot|\operatorname{Tr}(B g)| \\
& >(d(A, \mathscr{S})-\varepsilon) \cdot(d(B, \mathscr{T})-\varepsilon) .
\end{aligned}
$$

Since $h$ is a norm -1 operator in $\mathscr{S}_{\perp} \otimes \mathscr{T}_{\perp}$, and this is the preannihilator of $\mathscr{S} * \mathscr{T}$ by definition, this implies that $d(A \otimes B, \mathscr{S} * \mathscr{T})>(d(A, \mathscr{S})-\varepsilon) \cdot(d(B, \mathscr{T})-\varepsilon)$. Since $\varepsilon$ was arbitrary, the proof is complete.

Lemma 5. Let $\mathscr{S} \subseteq L(H)$ be a linear subspace, and let $x$ be a vector in $H \otimes K$. Let $F$ be the smallest projection in $L(H)$ such that $(F \otimes I) x=x$. Let $P$ be the orthogonal projection onto $[\mathscr{S} F H]$. Then $P \otimes I$ is the orthogonal projection onto $[(\mathscr{S} \otimes L(K)) x]$.

Proof. Let $\left\{e_{1}, e_{2}, \ldots\right\}$ be any orthonormal basis for $K$. Then there is a sequence $\left\{x_{i}\right\}$ of vectors in $H$ with $\sum\left\|x_{i}\right\|^{2}=\|x\|^{2}$ such that $x=\sum x_{i} \otimes e_{i}$. Let $E_{i}$ be the projection onto $\mathrm{C} e_{i}$. If $S \in \mathscr{S}, T \in L(K)$, then $\left(S \otimes T E_{i}\right) x=S x_{i} \otimes T e_{i}$. Hence $[(\mathscr{S} \otimes L(K)) x]$ contains all vectors of the form $S x_{i} \otimes y$ for arbitrary $S \in \mathscr{S}$. $y \in K$, for each $i$. Let $F^{\prime}$ be the projection onto the closed span of vectors $\left\{x_{i}\right.$ : $i=1,2, \ldots\}$. Then $F^{\prime} \geqslant F$, and we have

$$
\begin{aligned}
{[(\mathscr{S} \otimes L(K)) x] } & \supseteq\left[\mathscr{S} F^{\prime} H\right] \otimes K \supseteq[\mathscr{S} F H] \otimes K \\
& =[(\mathscr{S} \otimes L(K))(F H \otimes K)] \supseteq[(\mathscr{S} \otimes L(K)) x],
\end{aligned}
$$

so

$$
[(\mathscr{S} \otimes L(K)) x]=[\mathscr{S} F H] \otimes K=(P \otimes I)(H \otimes K) .
$$

Lemma 6. Let $H$ be a Hilbert space, let $\mathscr{S} \subseteq L(H)$ be a linear subspace, and let $h \in \mathscr{S}_{\perp}$ be a rank-1 operator. Then $h P=0$, where $P$ is the orthogonal projection onto $[\mathscr{S} h H]$.

ProOF. Write $h=v \otimes u$, where $u, v$ are vectors such that $h w=(w, v) u, w \in H$. Then $[\mathscr{S} h H]=[\mathscr{S} u]$. If $S \in \mathscr{S}$ we have $0=\operatorname{Tr}(S h)=(S u, v)$, so $[\mathscr{S} u] \perp v$; hence $P_{v}=0$. Then $h P=(P v) \otimes u=0$.

Lemma 7. Let $\mathscr{S} \subseteq L(H), \mathscr{T} \subseteq L(K)$ be linear subspaces, and let $\mathscr{R}=\mathscr{S} \otimes L(K)$ $+L(H) \otimes \mathscr{T}$. Let $x \in H \otimes K$. Let $F \in L(H), E \in L(K)$ be the smallest projections such that $F \otimes I$ and $I \otimes E$ contain $x$ in their range, and let $P$ be the projection onto $[\mathscr{S F H}]$ and $Q$ the projection onto $[\mathscr{T E K}]$. The projection onto $[\mathscr{R} x]$ is then $\left(P^{\perp} \otimes Q^{\perp}\right)^{\perp}$.

Proof. We have $[\mathscr{R} x]=[(\mathscr{S} \otimes L(K)) x] \vee[(L(H) \otimes \mathscr{T}) x]$. By Lemma 5 , the projections onto $[(\mathscr{S} \otimes L(K)) x]$ and $[(L(H) \otimes \mathscr{T}) x]$ are $P \otimes I$ and $I \otimes Q$, respectively. The projection onto $[\mathscr{R} x]$ is then $(P \otimes I) \vee(I \otimes Q)$, and since $P \otimes I$ and $I \otimes Q$ commute this reduces to $P \otimes I+I \otimes Q-P \otimes Q$. The orthogonal complement is then

$I \otimes I-P \otimes I-I \otimes Q+P \otimes Q=P^{\perp} \otimes I-P^{\perp} \otimes Q=P^{\perp} \otimes Q^{\perp}$, so $\operatorname{proj}[\mathscr{R} x]=\left(P^{\perp} \otimes Q^{\perp}\right)^{\perp}$. 
If $\mathscr{S}$ is a linear subspace of $L(H)$, we adopt the notation $\operatorname{ref}(\mathscr{S})$ to mean the smallest reflexive subspace of $L(H)$ containing $\mathscr{S}$. Thus $\operatorname{ref}(\mathscr{S})=\{T \in L(H)$ : $T x \in[\mathscr{S} x], x \in H\}$.

TheOREM 8. Let $\mathscr{S} \subseteq L(H), \mathscr{T} \subseteq L(K)$ be reflexive subspaces. Then $\mathscr{S} * \mathscr{T}$ is the smallest reflexive subspace containing $\mathscr{S} \otimes L(K)+L(H) \otimes \mathscr{T}$.

Proof. Let $\mathscr{R}=\mathscr{S} \otimes L(K)+L(H) \otimes \mathscr{T}$, and let $\hat{\mathscr{R}}=\operatorname{ref}(\mathscr{R})$. By definition, $\mathscr{R}$ and $\hat{\mathscr{R}}$ have the same closed cyclic subspaces. Since $\mathscr{S} * \mathscr{T}$ is reflexive and contains $\mathscr{R}$ it contains $\hat{R}$. To show equality, it will suffice to show that every rank-1 operator in $\hat{\mathscr{R}}_{\perp}$ is in $(\mathscr{S} * \mathscr{T})_{\perp}=\mathscr{S}_{\perp} \otimes \mathscr{T}_{\perp}$. Let $h$ be a rank-1 operator in $\mathscr{R}_{\perp}$, and let $x$ be a nonzero vector in the range of $h$. By Lemma $6, h=h G^{\perp}$, where $G$ is the projection onto $[\hat{\mathscr{R}} x]=[\mathscr{R} x]$. Let $F \in L(H), E \in L(K)$ be the smallest projections such that $(F \otimes I) x=x=(I \otimes E) x$, and let $P=\operatorname{proj}[\mathscr{S} F H], \quad Q=$ $\operatorname{proj}[\mathscr{T} E K]$. Then by Lemma $7, G^{\perp}=P^{\perp} \otimes Q^{\perp}$. We have $(F \otimes E) x=x$; hence

$$
h=(F \otimes E) h=(F \otimes E) h\left(P^{\perp} \otimes Q^{\perp}\right) \in(F \otimes E)\left(\mathscr{L}_{*}(H \otimes K)\right)\left(P^{\perp} \otimes Q^{\perp}\right),
$$

where $\mathscr{L}_{*}(H \otimes K)$ denotes the ideal of trace-class operators on $H \otimes K$. Since $\mathscr{L}_{*}(H \otimes K)$ is the trace-class norm closed span of elementary tensors $\{f \otimes g$ : $\left.f \in \mathscr{L}_{*}(H), g \in \mathscr{L}_{*}(K)\right\}$, the space $(F \otimes E)\left(\mathscr{L}_{*}(H \otimes K)\right)\left(P^{\perp} \otimes Q^{\perp}\right)$ is the closed span of elementary tensors $\left\{\left(F f P^{\perp}\right) \otimes\left(E g Q^{\perp}\right): f \in \mathscr{L}_{*}(H), g \in \mathscr{L}_{*}(K)\right\}$.

But for $f$ arbitrary and $S \in \mathscr{S}$ we have $\operatorname{Tr}\left(S F f P^{\perp}\right)=\operatorname{Tr}\left(P^{\perp} S F f\right)=0$ since $P^{\perp} \mathscr{S} F=0$. So $F f P^{\perp} \in S_{\perp}$. Similarly, $E g Q^{\perp} \in \mathscr{T}_{\perp}$ for all $g \in \mathscr{L}_{*}(K)$. So each $\left(F f P^{\perp}\right) \otimes\left(E g Q^{\perp}\right) \in \mathscr{S}_{\perp} \otimes \mathscr{T}_{\perp}$, and hence $h \in \mathscr{S}_{\perp} \otimes \mathscr{T}_{\perp}$.

THEOREM 9. Let $\mathscr{S} \subseteq L(H), \mathscr{T} \subseteq L(K)$ be reflexive proper subspaces. Then $\mathscr{K}(\mathscr{S} * \mathscr{T}) \geqslant \mathscr{K}(\mathscr{S}) \cdot \mathscr{K}(\mathscr{T})$.

Proof. Let $A \in L(H), B \in L(K)$ be arbitrary. By Proposition 4 we have $d(A \otimes B, \mathscr{S} * \mathscr{T})=d(A, \mathscr{S}) \cdot d(B, \mathscr{T})$. We will show that in general $\alpha(A \otimes$ $B, \mathscr{S} * \mathscr{T}) \leqslant \alpha(A, \mathscr{S}) \cdot \alpha(B, \mathscr{T})$, and hence if $A \notin \mathscr{S}$ and $B \notin \mathscr{T}$ then $\mathscr{K}(A \otimes$ $B, \mathscr{S} * \mathscr{T}) \geqslant \mathscr{K}(A, \mathscr{S}) \cdot \mathscr{K}(B, \mathscr{T})$. Taking of suprema over all such $A, B$ then yields $K(\mathscr{S} * \mathscr{T}) \geqslant \mathscr{K}(\mathscr{S}) \cdot \mathscr{K}(\mathscr{T})$, since by hypothesis $\mathscr{S} \neq L(H)$ and $\mathscr{T} \neq L(K)$.

We utilize Lemma 2. Let $x$ be a unit vector in $H \otimes K$. Let $\mathscr{R}=\mathscr{S} \otimes L(K)+$ $L(H) \otimes \mathscr{T}$. By Theorem $8, \mathscr{S} * \mathscr{T}=\operatorname{ref}(\mathscr{R})$, so $\mathscr{S} * \mathscr{T}$ and $\mathscr{R}$ have the same cyclic subspaces. As in the proof of Theorem 8 , let $G$ be the projection onto $[\mathscr{R} x]=$ $[(\mathscr{S} * \mathscr{T}) x]$, and let $F \in L(H), E \in L(K)$ be the smallest projections such that $(F \otimes I) x=(I \otimes E) x$. Let $P=\operatorname{proj}[\mathscr{S} F H], Q=\operatorname{proj}[\mathscr{T} E K]$. By Lemma $7, G=$ $\left(P^{\perp} \otimes Q^{\perp}\right)^{\perp}$. Since $(F \otimes E) x=x$ we have

$$
\begin{gathered}
d[(A \otimes B) x,(\mathscr{S} * \mathscr{T}) x]=\left\|G^{\perp}(A \otimes B) x\right\|=\left\|\left(P^{\perp} \otimes Q^{\perp}\right)(A \otimes B)(F \otimes E) x\right\| \\
=\left\|\left(\left(P^{\perp} A F\right) \otimes\left(Q^{\perp} B E\right)\right) x\right\| \leqslant\left\|P^{\perp} A F\right\| \cdot\left\|Q^{\perp} B E\right\| \leqslant \alpha(A, \mathscr{S}) \cdot \alpha(B, \mathscr{T})
\end{gathered}
$$

since $P^{\perp} \mathscr{S} F=0$ and $Q^{\perp} \mathscr{T} E=0$.

Since $x$ was an arbitrary unit vector, we have

$$
\alpha(A \otimes B, \mathscr{S} * \mathscr{T}) \leqslant \alpha(A, \mathscr{S}) \cdot \alpha(B, \mathscr{T}) \text {, as required. }
$$


The proof of Theorem 9 can be improved slightly to show that for arbitrary $A \in L(H), B \in L(K)$ the inequality $\alpha(A \otimes B, \mathscr{S} * \mathscr{T}) \leqslant \alpha(A, \mathscr{S}) \cdot \alpha(B, \mathscr{T})$ is in fact an equality. We capture this fact.

Corollary 10. Let $\mathscr{S} \subseteq L(H)$ and $\mathscr{T} \subseteq L(K)$ be reflexive subspaces, and let $A \in L(H), B \in L(K)$ be arbitrary. Then $\alpha(A \otimes B, \mathscr{S} * \mathscr{T})=\alpha(A, \mathscr{S}) \cdot \alpha(B, \mathscr{T})$.

Proof. The inequality " $\leqslant$ " is contained in the proof of Theorem 9. For the converse, let $F, P \in L(H)$ and $E, Q \in L(K)$ be arbitrary projections satisfying $P^{\perp} \mathscr{S} F=0$ and $Q^{\perp} \mathscr{T} E=0$. Then if $\mathscr{R}=\mathscr{S} \otimes L(K)+L(H) \otimes \mathscr{T}$, we have $\left(P^{\perp} \otimes Q^{\perp}\right) \mathscr{R}(F \otimes E)=0$, so since $\mathscr{S} * \mathscr{T}=\operatorname{ref}(\mathscr{R})$ by Theorem 8 , we have $\left(P^{\perp} \otimes Q^{\perp}\right)(\mathscr{S} * \mathscr{T})(F \otimes E)=0$. Since $\left\|\left(P^{\perp} \otimes Q^{\perp}\right)(A \otimes B)(F \otimes E)\right\|=\left\|P^{\perp} A F\right\|$ $\cdot\left\|Q^{\perp} B E\right\|$, we have

$\alpha(A \otimes B, \mathscr{S} * \mathscr{T})=\sup \{\|L(A \otimes B) M\|: L, M$ are projections

$$
\begin{aligned}
& \text { in } L(H \otimes K) \text { with } L(\mathscr{S} \otimes \mathscr{T}) M=0\} \\
& \geqslant\left\|P^{\perp} A F\right\| \cdot\left\|Q^{\perp} B E\right\| .
\end{aligned}
$$

So since $P, F, Q, E$ were arbitrary, we conclude that $\alpha(A \otimes B, \mathscr{S} * \mathscr{T}) \geqslant \alpha(A, \mathscr{S})$ - $\alpha(B, \mathscr{T})$. Finally, we note that equality is trivially true if either $\mathscr{S}=L(H)$ or $\mathscr{T}=L(K)$.

From Corollary 10 and Proposition 4 we conclude that $\mathscr{K}(A \otimes B, \mathscr{S} * \mathscr{T})=$ $\mathscr{K}(A, \mathscr{S}) \cdot \mathscr{K}(B, \mathscr{T})$ whenever $\mathscr{S}, \mathscr{T}$ are reflexive proper subspaces with $A \notin \mathscr{S}$, $B \notin \mathscr{T}$. That is, the basic inequality is an equality when restricted to the class of elementary tensors. It can happen, however, that for some operator $T \in L(H \otimes K)$, which is not an elementary tensor, we have $\mathscr{K}(T, \mathscr{S} * \mathscr{T})>\mathscr{K}(\mathscr{S}) \cdot \mathscr{K}(\mathscr{T})$, and thus the inequality in Theorem 9 may be strict. The following simple example shows this.

EXAMPLE 11. Let

$$
S=\left(\begin{array}{ll}
* & 0 \\
0 & 0
\end{array}\right)=\left\{\left(\begin{array}{ll}
\lambda & 0 \\
0 & 0
\end{array}\right): \lambda \in \mathbf{C}\right\}
$$

be regarded as a subspace of operators acting on 2-dimensional Hilbert space. An elementary computation shows that $S$ is reflexive. An application of [6, Lemma 3.3] after interchanging rows, and either [4, Proposition 3 or $\mathbf{5}$, Theorems 1.1 or 1.2] to the preannihilator

$$
S_{\perp}=\left(\begin{array}{cc}
0 & * \\
* & *
\end{array}\right)
$$

shows that $\mathscr{K}(S)=1$. Since

$$
\mathscr{S}_{\perp} \otimes \mathscr{S}_{\perp}=\left(\begin{array}{cccc}
0 & 0 & 0 & * \\
0 & 0 & * & * \\
0 & * & 0 & * \\
* & * & * & *
\end{array}\right)
$$


we have

$$
S * S=\left(\begin{array}{cccc}
* & * & * & 0 \\
* & * & 0 & 0 \\
* & 0 & * & 0 \\
0 & 0 & 0 & 0
\end{array}\right)
$$

acting on 4-dimensional Hilbert space. Let

$$
P=\left(\begin{array}{llll}
0 & 0 & 0 & 0 \\
0 & 1 & 0 & 0 \\
0 & 0 & 1 & 0 \\
0 & 0 & 0 & 1
\end{array}\right) .
$$

Then $P(S * S) \subseteq S * S$, and thus by [5, Lemma 1.3] the compression $\left.P(S * S)\right|_{P H}$ is reflexive with hyperreflexivity constant no greater than that of $S * S$. But this compression has diagram

$$
\left(\begin{array}{lll}
* & 0 & 0 \\
0 & * & 0 \\
0 & 0 & 0
\end{array}\right)
$$

and by [5, Example 4.7] this has constant $\geqslant \sqrt{9 / 8}$. Thus $\mathscr{K}(S * S) \geqslant \sqrt{9 / 8}>1=$ $\mathscr{K}(S) \cdot \mathscr{K}(S)$. So in this case the inequality of Theorem 9 is strict.

THEOREM 12. Let $n$ be a positive integer, and for $1 \leqslant i \leqslant n$ let $\mathscr{A}_{i}$ be a reflexive proper subalgebra of $L\left(H_{i}\right)$ for $H_{i}$ a separable Hilbert space. Suppose the tensor product $\mathscr{A}_{1} \otimes \cdots \otimes \mathscr{A}_{n}$, acting on $H=H_{1} \otimes \cdots \otimes H_{n}$, is reflexive. Let

$$
\mathscr{A}=\left(\begin{array}{cc}
\mathscr{A}_{1} \otimes \cdots \otimes \mathscr{A}_{n} & \mathscr{A}_{1} * \cdots * \mathscr{A}_{n} \\
0 & \mathscr{A}_{1} \otimes \cdots \otimes \mathscr{A}_{n}
\end{array}\right) .
$$

Then $\mathscr{A}$ is a reflexive subalgebra of $L(H \otimes H)$, and $\mathscr{K}(\mathscr{A}) \geqslant \mathscr{K}\left(\mathscr{A}_{1}\right) \cdot \mathscr{K}\left(\mathscr{A}_{2}\right) \cdots$ $\mathscr{K}\left(\mathscr{A}_{n}\right)$.

PROOF. $\mathscr{A}$ is an algebra since $\mathscr{A}_{1} * \cdots * \mathscr{A}_{n}$ is a bimodule over $\mathscr{A}_{1} \otimes \cdots \otimes \mathscr{A}_{n}$. A simple calculation shows that

$$
\mathscr{A}_{\perp}=\left(\begin{array}{cc}
\left(\mathscr{A}_{1} \otimes \cdots \otimes \mathscr{A}_{n}\right)_{\perp} & L_{*}(H) \\
\left(\mathscr{A}_{1} * \cdots * \mathscr{A}_{n}\right)_{\perp} & \left(\mathscr{A}_{1} \otimes \cdots \otimes \mathscr{A}_{n}\right)_{\perp}
\end{array}\right) .
$$

Since $\mathscr{A}_{1} \otimes \cdots \otimes \mathscr{A}_{n}$ is reflexive, $\left(\mathscr{A}_{1} \otimes \cdots \otimes \mathscr{A}_{n}\right)_{\perp}$ is generated by rank-1 operators. Since $\mathscr{A}_{1} \cdots \mathscr{A}_{n}$ are reflexive, $\left(\mathscr{A}_{1} * \cdots * \mathscr{A}_{n}\right)_{\perp}=\left(\mathscr{A}_{1}\right)_{\perp} \otimes \cdots \otimes\left(\mathscr{A}_{n}\right)_{\perp}$ is also generated by rank-1 operators. Hence $\mathscr{A}_{\perp}$ is generated by rank-1 operators, so $\mathscr{A}$ is reflexive.

To show $\mathscr{K}(\mathscr{A}) \geqslant \mathscr{K}\left(\mathscr{A}_{1}\right) \cdots \mathscr{K}\left(\mathscr{A}_{n}\right)$ we utilize [4, Proposition 3]. Let $P$ be the orthogonal projection from $H \oplus H$ onto $H$. Let $S=\mathscr{A}_{1} * \cdots * \mathscr{A}_{n}$. By Theorem 9, $\mathscr{K}(S) \geqslant \mathscr{K}\left(\mathscr{A}_{1}\right) \cdots \mathscr{K}\left(\mathscr{A}_{n}\right)$. Let $\mathscr{C}_{1}(\mathscr{A}), \mathscr{C}_{1}(S)$ denote the closed convex hulls of the rank $\leqslant 1$ operators in the unit balls of $\mathscr{A}_{\perp}, S$, respectively. Then clearly

$$
P \perp \mathscr{C}_{1}(\mathscr{A}) P=\left(\begin{array}{cc}
0 & 0 \\
\mathscr{C}_{1}(S) & 0
\end{array}\right) \text {. }
$$


Let $R(\mathscr{A}), R(S)$ be the largest radii such that $\left\{f \in \mathscr{A}_{\perp}:\|f\|_{1} \leqslant R(\mathscr{A})\right\} \subseteq \mathscr{C}_{1}(\mathscr{A})$ and $\left\{g \in S_{\perp}:\|g\|_{1} \leqslant R(S)\right\} \subseteq \mathscr{C}_{1}(S)$. It follows that $R(\mathscr{A}) \leqslant R(S)$. By [4, Proposition 3] we have $\mathscr{K}(\mathscr{A})=1 / R(\mathscr{A})$ and $\mathscr{K}(S)=1 / R(S)$, so $\mathscr{K}(\mathscr{A}) \geqslant$ $\mathscr{K}(S)$, as required.

Remarks. The requirement that $\mathscr{A}_{1} \otimes \cdots \otimes \mathscr{A}_{n}$ be reflexive will be met if each $\mathscr{A}_{i}$ is finite dimensional, and more generally, if each $\mathscr{A}_{i}$ has property $S_{\sigma}$ (Kraus [3]). (It is, of course, an open question whether the tensor product of reflexive algebras is necessarily reflexive.) As in the special case of the "key example" in [2], Theorem 12 gives a means of constructing reflexive algebras of arbitrarily large distance constant. If each $\mathscr{A}_{i}$ is a CSL algebra and so contains a m.a.s.a., then $\mathscr{A}$ will also contain a m.a.s.a., so will be a CSL algebra. A direct sum of such algebras, with increasing constants, will be nonhyperreflexive.

\section{REFERENCES}

1. W. Arveson, Ten lectures on operator algebras, CBMS Regional Conf. Ser. in Math., No. 55, Amer. Math. Soc., Providence, R.I., 1984.

2.K. Davidson and S. Power, Failure of the distance formula, preprint.

3. J. Kraus, Tensor products of reflexile algebras, J. London Math. Soc. (2) 28 (1983), 350-358.

4. J. Kraus and D. Larson, Some applications of a technique for constructing reflexice operator algebras, J. Operator Theory 13 (1985), 227-236.

5. Reflexivitty and distance formulae, Proc. London Math. Soc. (to appear).

6. E. C. Lance, Cohomology and perturhations of nest algebras. Proc. London Math. Soc. 43 (1981). $334-356$.

7. D. Larson, Annihilators of operators algebras, Topics in Modern Operator Theory, vol. 6, Birkhauser. Basel, 1982, pp. 119-130.

Department of Mathematics and Statistics, University of Nebraska. Lincoln. Nebraska 68588 